\title{
Electron Energy-Loss Cross Sections for the Chemical Bond Overlap Plasmon of the Hydrogen Molecule
}

\author{
Oscar L. Malta, Renaldo T. Moura Jr.* and Ricardo L. Longo \\ Departamento de Química Fundamental - CCEN, Universidade Federal de Pernambuco, \\ Cidade Universitária, 50740-540 Recife-PE, Brazil
}

\begin{abstract}
Examinamos a possibilidade de detectar o plásmon da região de recobrimento da ligação química (CBOP) da molécula de hidrogênio através do espalhamento inelástico de elétrons. Foi previsto que o CBOP absorve e espalha eficientemente a radiação eletromagnética acima do limiar de ionização molecular para o caso dos halogenetos de metais alcalinos. Para a molécula de hidrogênio, a natureza quadrupolar da seção de choque do CBOP fornece valores de seção de choque para perda de energia que dependem da energia do feixe de elétrons incidente e um comportamento angular que é totalmente distinto da ionização usual, das transições inter-bandas e dos processos dissociativos. Algumas relações obtidas anteriormente entre o CBOP e a polarizabilidade da região de recobrimento sugerem ser essa grandeza uma ferramenta teórica promissora na quantificação da covalência em ligações químicas.
\end{abstract}

We examine the possibility of detecting the chemical bond overlap plasmon (CBOP) of the hydrogen molecule by electron inelastic scattering. The CBOP has been predicted to efficiently absorb and scatter electromagnetic radiation above the molecular ionization threshold in the cases of alkali halides. For the hydrogen molecule the quadrupole nature of the CBOP energy-loss cross section leads to cross section values with impacting electron energy dependence and an angular behavior which are totally distinguishable from the usual ionization, inter-band transitions and dissociation processes. Previously established relationships between the CBOP and the polarizability of the overlap region suggest this an a promising theoretical tool for quantifying covalency in the chemical bond.

Keywords: energy loss, bond overlap plasmon, $\mathrm{H}_{2}$, electron scattering, covalency

\section{Introduction}

In a relatively recent attempt to obtain a deeper insight on covalency in lanthanide compounds, the concepts of chemical bond overlap polarizability (OP) and ionic specific valence (ISV) have been introduced. ${ }^{1}$ This has allowed the definition of a covalency scale and a description of ligand fields in coordination compounds in terms of a potential that may be treated non phenomenologically when charge factors, appearing in the simple overlap model, ${ }^{2}$ are identified with the ligating atoms ISV's. As far as each pair lanthanide ion-ligating atom is considered as a diatomiclike molecule, these charge factors are a simple function of the chemical bond force constant, internuclear distance and LUMO-HOMO energy difference.

\footnotetext{
*e-mail: renaldotmjr@gmail.com
}

The OP and ISV concepts have also been explored in a more general context outside the scope of ligand field theory. They have proven to be useful in the case of diatomic molecules, allowing to establish a new covalency scale in excellent agreement with Pauling's scale ${ }^{3,4}$ and analytically quantifiable in terms of the OP. Relevant questions could be raised on possible relationships between macroscopic properties of materials and these concepts. Thus, for example, a good correlation has been found between the non-linear index of refraction $\left(\mathrm{n}_{2}\right)$ and the OP. ${ }^{4}$ More recently, a proposal in which the overlap region is regarded as a localized plasmon-like charge distribution (chemical bond overlap plasmon-CBOP), characterized by the OP, has raised the question on the possibility of absorption and inelastic scattering of radiation by the overlap region, above the first ionization threshold. ${ }^{5}$ Predicted oscillator strengths and scattering cross sections for diatomic molecules are considerably high and can be measured in the UV and near 
soft-X-rays spectral regions. An interesting aspect is that, formally, oscillation modes corresponding to $\sigma$ and $\pi$ bonds can be distinguished by these processes, on the grounds of their quite distinct electronic clouds as, for instance, in the carbon monoxide molecule.

In the present work we calculate, within a Born-type approximation, the electron energy-loss cross section for the $\mathrm{CBOP}$ of the $\mathrm{H}_{2}$ molecule as a function of the incident electron energy and scattering angle. The aim is to examine how this inelastic scattering cross section compares with the cross section values for processes involving bound-tobound (inter-band) transitions, ionization and dissociation. An interesting feature of the present model and calculations is that no molecular continuum wave functions are involved.

\section{Results and Discussion}

\section{Electron energy-loss cross sections for the CBOP}

\section{Chemical bond overlap plasmon}

The CBOP theory has been developed in reference 1 as a consequence of the concepts of overlap polarizability and ionic specific valence introduced in reference 1 . In this subsection we briefly present the main points of the theory and their application to the case of the $\mathrm{H}_{2}$ molecule.

The overlap polarizability, $\alpha_{\mathrm{OP}}$, is given by ${ }^{1}$

$\alpha_{\mathrm{OP}}=\frac{\mathrm{e}^{2} \rho^{2} \mathrm{R}^{2}}{2 \Delta \mathrm{E}}$

where

$\rho^{2}=\sum_{\xi, \zeta} S_{\xi, \zeta}^{2}$

with $S_{\xi, \zeta}$ being the overlap integral between orbitals $|\xi\rangle$ and $|\zeta\rangle$ involved in the formation of a given chemical bond. Distinguishing between $\sigma$ and $\pi$ bonds is a matter of taking the appropriate sum over valence shell orbitals in equation 2 for each case. $\mathrm{R}$ is the internuclear distance, $\Delta \mathrm{E}$ is the energy difference between the LUMO and HOMO associated with the chemical bond, and e is the elementary charge.

The overlap charge, q, is postulated to satisfy the following relations:

$\mathrm{q}^{2}=\mathrm{k} \alpha_{\mathrm{OP}}$

and

$q=v \rho e$

where $\mathrm{k}$ is the force constant of the chemical bond and the ionic specific valence, $\mathrm{v}$, is given by

$$
\mathrm{v}=\mathrm{R} \sqrt{\frac{\mathrm{k}}{2 \Delta \mathrm{E}}}
$$

The theory also postulates that the CBOP excitation frequency, $\omega_{0}$, is given by the harmonic oscillator behavior according to

$\omega_{0}=\sqrt{\frac{\mathrm{k}}{\mathrm{m}}}$

where

$\mathrm{m}=\mathrm{v} \rho \mathrm{m}_{\mathrm{e}}$

and $\mathrm{m}_{\mathrm{e}}$ being the electron mass. Thus, the CBOP may act as an open scattering channel.

\section{Electron energy-loss cross sections}

The inelastic scattering process by the CBOP is schematized in Figure 1. An incident electron on the $\mathrm{z}$ axis, with wave vector $\overrightarrow{\mathrm{K}}$, hits a target molecule, considered as a spherical system located at the origin of the reference frame, and is scattered with a wave vector $\overrightarrow{\mathrm{K}}^{\prime}$. From the addition theorem for spherical harmonics the interaction Hamiltonian between an incident electron and the CBOP is given by

$\mathrm{H}_{\text {int }}=\operatorname{vpe}^{2} \sum_{\mathrm{t}, \mathrm{p}}\left(\frac{4 \pi}{2 \mathrm{t}+1}\right) \frac{\mathrm{r}^{\mathrm{t}}}{\mathrm{r}^{\mathrm{t}+1}} \mathrm{Y}_{\mathrm{t}, \mathrm{p}}^{*}\left(\Omega^{\prime}\right) \mathrm{Y}_{\mathrm{t}, \mathrm{p}}(\Omega)$

where the position vector $\vec{r}$ ' stands for the overlap region and $\vec{r}$ stands for the impacting electron. It is assumed that r' $<$ r.

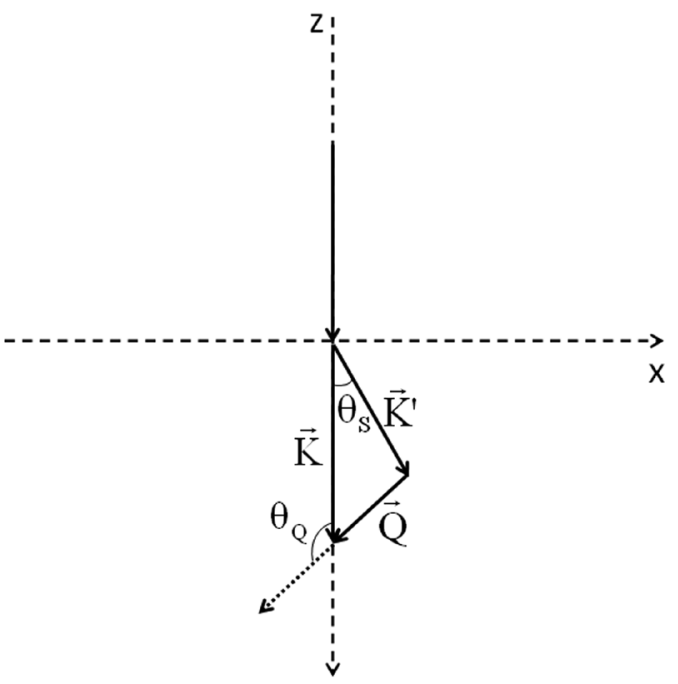

Figure 1. Inelastic scattering process and the definitions of the coordinate $\operatorname{system}(\mathrm{x}, \mathrm{z})$, the wave vectors $(\overrightarrow{\mathrm{K}}, \overrightarrow{\mathrm{K}}, \overrightarrow{\mathrm{Q}})$ and the scattering angles $\left(\theta_{\mathrm{S}}, \theta_{\mathrm{Q}}\right)$. 
The scattering cross section, in a unitary interaction volume, per scattering solid angle is given by ${ }^{6}$

$\frac{\mathrm{d} \sigma}{\mathrm{d} \Omega_{\mathrm{s}}}=\frac{\mathrm{m}_{\mathrm{e}}^{2}}{\left(2 \pi \hbar^{2}\right)^{2}} \frac{\mathrm{K}^{\prime}}{\mathrm{K}}\left|\left\langle\psi^{\prime}\left|\mathrm{H}_{\text {int }}\right| \psi\right\rangle\right|^{2}$

where the total initial and final wave functions are expressed as

$|\psi\rangle=|0\rangle \mathrm{e}^{\mathrm{i} \overrightarrow{\mathrm{K}} \cdot \overrightarrow{\mathrm{r}}}$

and

$\left|\psi^{\prime}\right\rangle=|1\rangle \mathrm{e}^{\mathrm{i} \overrightarrow{\mathrm{K}}^{\prime} \cdot \overrightarrow{\mathrm{r}}}$

where $|0\rangle$ and $|1\rangle$ indicate the initial and final state of the CBOP. Conservation of energy requires that

$\frac{\hbar^{2} \mathrm{~K}^{2}}{2 \mathrm{~m}_{\mathrm{e}}}=\frac{\hbar^{2} \mathrm{~K}^{\prime 2}}{2 \mathrm{~m}_{\mathrm{e}}}+\hbar \omega_{0}$

$\hbar \omega_{0}$ being the CBOP energy.

Since the dipole moment of the excited CBOP vanishes for $\mathrm{A}_{2}$-type molecules, the relevant term in the expansion in equation 8 corresponds to $t=2$ (the quadrupole term). The matrix element in equation 9 reduces to

$\left\langle\psi^{\prime}\left|\mathrm{H}_{\text {int }}\right| \psi\right\rangle=\operatorname{vpe}^{2} \frac{4 \pi}{5} \sum_{\mathrm{p}}\left\langle 1\left|\mathrm{r}^{2} \mathrm{Y}_{2, \mathrm{p}}^{*}\left(\Omega^{\prime}\right)\right| 0\right\rangle \int_{\frac{\mathrm{e}^{\mathrm{i}} \overrightarrow{\mathrm{Q}} \cdot \overrightarrow{\mathrm{r}}}{\mathrm{r}^{3}}}^{\mathrm{r}_{2, \mathrm{p}}}(\Omega) \mathrm{r}^{2} \mathrm{drd} \Omega$

where $\vec{Q}=\vec{K}-\vec{K}$ '. Using the partial waves expansion

$\mathrm{e}^{\mathrm{i} \overrightarrow{\mathrm{Q}} \cdot \overrightarrow{\mathrm{r}}}=4 \pi \sum_{\ell, \mathrm{m}_{\ell}} \mathrm{i}^{\ell} \mathrm{j}_{\ell}(\mathrm{Qr}) \mathrm{Y}_{\ell, \mathrm{m}_{\ell}}^{*}(\Omega) \mathrm{Y}_{\ell, \mathrm{m}_{\ell}}\left(\Omega_{\mathrm{Q}}\right)$

where $\mathrm{j}_{\ell}(\mathrm{Qr})$ is a spherical Bessel's function, equation 13 becomes

$\left\langle\psi^{\prime}\left|\mathrm{H}_{\text {int }}\right| \psi\right\rangle=-v \rho e^{2} \frac{16 \pi^{2}}{5} \sum_{\mathrm{p}}\left\langle 1\left|\mathrm{r}^{\prime 2} \mathrm{Y}_{2, \mathrm{p}}^{*}\left(\Omega^{\prime}\right)\right| 0\right\rangle \mathrm{Y}_{2, \mathrm{p}}\left(\Omega_{\mathrm{Q}}\right) \int_{0}^{\infty} \frac{\mathrm{j}_{2}(\mathrm{Qr})}{\mathrm{r}} \mathrm{dr}$

The orthonormality relation for the spherical harmonics has been used in the above equation. An interesting feature is that the radial integral involving the spherical Bessel's function in this equation is independent of $\mathrm{Q}$, having a value equal to $1 / 3$. Evaluation of the matrix element given by equation 15 is not an easy task due to the difficulty in evaluating the p-spherical component of the quadrupole transition moment of the CBOP. This difficulty can be considerably facilitated if this p-dependent quadrupole transition moment is replaced by an average quadrupole moment of the CBOP, $\mathbf{M}^{(2)}$. This approximation is better justified on the basis of the rotational degrees of freedom of the target molecule. Thus, equation 15 may be approximated as

$$
\left\langle\psi^{\prime}\left|\mathrm{H}_{\mathrm{int}}\right| \psi\right\rangle=-\mathrm{vpe}^{2} \frac{16 \pi^{2}}{15} \mathrm{M}^{(2)} \sum_{\mathrm{p}} \mathrm{Y}_{2, \mathrm{p}}\left(\Omega_{\mathrm{Q}}\right)
$$

The quadrupole contribution to the differential cross section in equation 9 is then given by

$\frac{\mathrm{d} \sigma}{\mathrm{d} \Omega_{\mathrm{s}}}=\left(\frac{8 \pi}{15}\right)^{2}\left(\frac{\mathrm{vpm} \mathrm{e}^{2}}{\hbar^{2}}\right)^{2}\left(\frac{\mathrm{E}-\hbar \omega_{0}}{\mathrm{E}}\right)^{1 / 2}\left(\mathrm{M}^{(2)}\right)^{2} \sum_{\mathrm{p}, \mathrm{p}^{\prime}} \mathrm{Y}_{2, \mathrm{p}}\left(\Omega_{\mathrm{Q}}\right) \mathrm{Y}_{2, \mathrm{p}^{*}}^{*}\left(\Omega_{\mathrm{Q}}\right)($

where $\mathrm{E}$ is the kinetic energy of the incident electron.

The angular dependence of the r.h.s. of equation 17 is better analyzed if the following relation is used: ${ }^{7}$

$$
\begin{array}{r}
\sum_{\mathrm{p}, \mathrm{p}^{\prime}} \mathrm{Y}_{2, \mathrm{p}}\left(\Omega_{\mathrm{Q}}\right) \mathrm{Y}_{2, \mathrm{p}^{\prime}}^{*}\left(\Omega_{\mathrm{Q}}\right)=5 \sum_{\mathrm{p}, \mathrm{p}^{\prime}, \ell \mathrm{m}_{\ell}}(-1)^{\mathrm{p}^{\prime}}\left(\frac{2 \ell+1}{4 \pi}\right)^{1 / 2} \\
\left(\begin{array}{rrl}
2 & 2 & \ell \\
-\mathrm{p}^{\prime} & \mathrm{p} & \mathrm{m}_{\ell}
\end{array}\right)\left(\begin{array}{lll}
2 & 2 & \ell \\
0 & 0 & 0
\end{array}\right) \mathrm{Y}_{\ell, \mathrm{m}_{\ell}}^{*}\left(\Omega_{\mathrm{Q}}\right)
\end{array}
$$

where ( ) is a $3-\mathrm{j}$ symbol. The cross section is totally symmetric with respect to the azimuthal angle $\phi_{\mathrm{Q}}$. Therefore, the r.h.s. of equation 17 may be replaced by an average over $\phi_{\mathrm{Q}}$ and, from equation 18, it is not difficult to see that the summation over the product of spherical harmonics reduces to $5 / 4 \pi$, giving

$\frac{\mathrm{d} \sigma}{\mathrm{d} \Omega_{\mathrm{s}}}=\left(\frac{8 \pi}{15}\right)^{2}\left(\frac{\mathrm{v \rho m}_{\mathrm{e}} \mathrm{e}^{2}}{\hbar^{2}}\right)^{2}\left(\frac{\mathrm{E}-\hbar \omega_{0}}{\mathrm{E}}\right)^{1 / 2}\left(\mathrm{M}^{(2)}\right)^{2} \frac{5}{4 \pi}$

The total cross section in a scattering solid angle specified by the position and characteristics of the detection instrument is then given by

$\sigma=\left(\frac{8 \pi}{15}\right)^{2}\left(\frac{\mathrm{v \rho m}_{\mathrm{e}} \mathrm{e}^{2}}{\hbar^{2}}\right)^{2}\left(\frac{\mathrm{E}-\hbar \omega_{0}}{\mathrm{E}}\right)^{1 / 2}\left(\mathrm{M}^{(2)}\right)^{2} \frac{5}{4 \pi} \Delta \phi \int_{\theta_{1}}^{\theta_{2}} \sin (\theta) \mathrm{d} \theta$

The distance from the target molecule to the detection instrument and its aperture define the angles $\Delta \phi=\phi_{2}-\phi_{1}$, $\theta_{1}$ and $\theta_{2}$. This cross section has a maximum for a scattering angle, $\theta_{\mathrm{s}}$ (between $\theta_{1}$ and $\theta_{2}$ ), equal to $\pi / 2$.

\section{Numerical estimates}

In order to discuss the important features of the cross section given by equation 20 an estimate of the CBOP average quadrupole moment is needed. The quadrupole moment of the $\mathrm{H}_{2}$ molecule has been estimated to be $0.22 \AA^{2.8}$. Thus, it is reasonable to assume (in $\mathrm{cm}^{2}$ ) that

$\mathrm{M}^{(2)}=0.22 \times 10^{-16} \rho$

The overlap integral between the two $1 s$ orbitals is expressed as, ${ }^{9}$ 
$\rho(R)=\left(1+\zeta R+\zeta^{2} R^{2} / 3\right) e^{-\zeta R}$

for $\mathrm{R}$ in $a_{0}$ and with $\zeta$ being the orbital exponent in $a_{0}^{-1}$. Using $\mathrm{R}=0.74 \AA=1.40 a_{0}$ and $\zeta=1.0 a_{0}^{-1}$ we find $\rho=0.753$. Considering the force constant, $\mathrm{k}=5.1 \times 10^{5}$ dyne $\mathrm{cm}^{-1},{ }^{10}$ and $\mathrm{v}=1$, the CBOP excitation energy, from equations 6 and 7, is found to be $18 \mathrm{eV}$, which is $c a .2 .5 \mathrm{eV}$ above the ionization threshold of the $\mathrm{H}_{2}$ molecule. This result should be considered as a lower bound value, since if the orbital exponent is optimized, ${ }^{11}$ at the equilibrium distance, $1.42 a_{0}$, it is equal to $1.17 a_{0}^{-1}$, so that $\rho=0.680$ and the CBOP excitation energy becomes $19 \mathrm{eV}$. In addition, the valence bond treatment can be improved by including the ionic contribution $\left(\psi_{\mathrm{I}}\right)$ to the Heitler-London covalent function $\left(\psi_{\mathrm{C}}\right)$. Indeed, if the covalent and ionic functions are non-orthogonal, the optimized wavefunction at the energy minimum (optimum $\zeta$ ) is ${ }^{9}$

$\Psi=0.801981 \psi_{\mathrm{C}}+0.211702 \psi_{\mathrm{I}}$

If the ionic contribution is orthogonalized to the covalent function, this wavefunction becomes

$\Psi=0.99650 \psi_{\mathrm{C}}+0.08354 \psi_{\mathrm{I}}^{\prime}$

In any case, there is an ionic contribution to the wavefunction when localized (optimum) atomic orbitals are used. Thus, the ionic specific valence, $\mathrm{v}$, should be smaller than 1.0, which leads to an increase of the CBOP excitation energy. For instance, for non-orthogonal functions the ionic contribution is nearly $5 \%$ so that $\mathrm{v} c a$. 0.95 which yields a CBOP excitation energy $c a .19 .5 \mathrm{eV}$. Ascribing $\triangle \mathrm{E}$ in equation 5 as the first excitation energy of $\mathrm{H}_{2}$, namely $\Delta \mathrm{E} c a .12 .7 \mathrm{eV}$ yields a value of 0.82 for the ionic specific valence, according to equation 5 , and thus a value of $c a .21 \mathrm{eV}$ for the CBOP excitation energy. As a result, a good estimate of the CBOP excitation energy would be $20 \pm 1 \mathrm{eV}$. We performed TDHF/aug-ccpVQZ calculations $^{12}$ for $\mathrm{H}_{2}$ at $0.736 \AA$ and found some highly excited states at 18.9, 20.2 and $24.0 \mathrm{eV}$. All these states have a Rydberg character, thus having a very distinct electronic density from the CBPO charge density. It is thus expected that, in despite of the CBOP excitation energy being in the energy range of highly excited discrete states they should have very distinct angular dependence in the differential cross section electron energy loss spectra.

The cross section in equation 20 presents interesting and distinguishable features in comparison with electron energy-loss cross sections for usual processes like interband transitions, ionization and dissociation. The first aspect to be noted is that the differential cross section $\left(\mathrm{d} \sigma / \mathrm{d} \Omega_{\mathrm{s}}\right)$ in equation 19 assumes values between 0.023 and 0.071 , in units of $\pi a_{0}^{2}$, for incident electron energies between $20 \mathrm{eV}$ and $1000 \mathrm{eV}$, respectively. These values are basically of the same order as the ones estimated, both experimentally and theoretically, for the usual processes mentioned above. ${ }^{13,14}$ They are only a factor of three smaller then the values for ionization at these two energies. ${ }^{14}$ The first distinguishable aspect to be noted is the energy dependence presented by equation 19 . In contrast to the cases of inter-band, ionization and dissociation processes, the CBOP differential cross section presents no maximum; it increases with increasing incident energy and goes asymptotically to a value around 0.07 (in units of $\pi a_{0}^{2}$ ), as shown in Figure 2. This behavior is a consequence of the quadrupole nature of the electron scattering process by the $\mathrm{CBOP}$ in $\mathrm{A}_{2}$-type molecules.

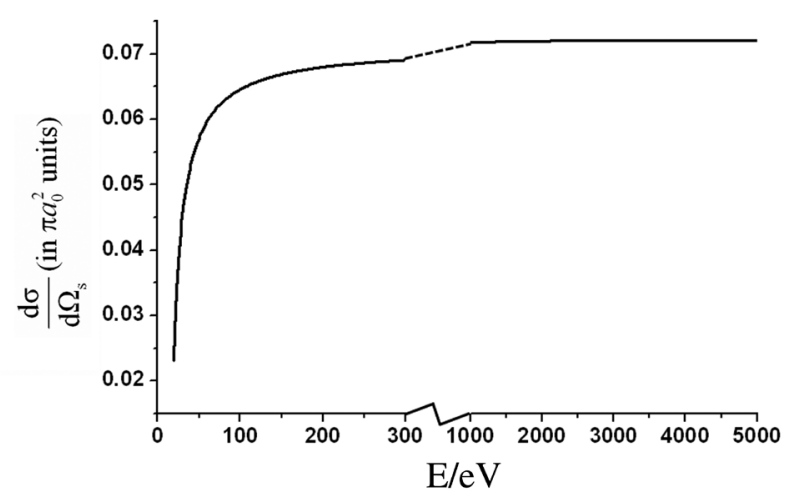

Figure 2. Dependence of the energy loss differential cross section $\left(\mathrm{d} \sigma / \mathrm{d} \Omega_{\mathrm{s}}\right)$ with incident energy $(\mathrm{E})$.

The second aspect is the angular dependence presented by the total cross section given by equation 20 . The sinusoidal angular dependence is predicted to be independent of the incident energy, also in contrast to the inter-band, ionization and dissociation cases. In these cases, both the differential and total cross sections have a maximum between $15 \mathrm{eV}$ and $100 \mathrm{eV}$, and decrease as the incident energy increases, with angular dependence peaked between $30^{\circ}$ and $40^{\circ},{ }^{13}$ while in the $\mathrm{CBOP}$ case it is peaked at $90^{\circ}$. The angular dependence of the total cross section is depicted in Figure 3 for an incident energy equals to $1000 \mathrm{eV}$, aperture angular resolutions $\Delta \phi=5^{\circ}$ (fixed) and $\Delta \theta=5^{\circ}$ with $\theta$ varying from $0^{\circ}$ to $180^{\circ}$ and plotted as a function of the final angle $\theta_{2}$.

\section{Conclusions}

Once the $\mathrm{CBOP}$ is directly related to the polarizability of the overlap region, which in turn is directly associated to the degree of covalency, its assessment by means of spectroscopic techniques may constitute a tool for 


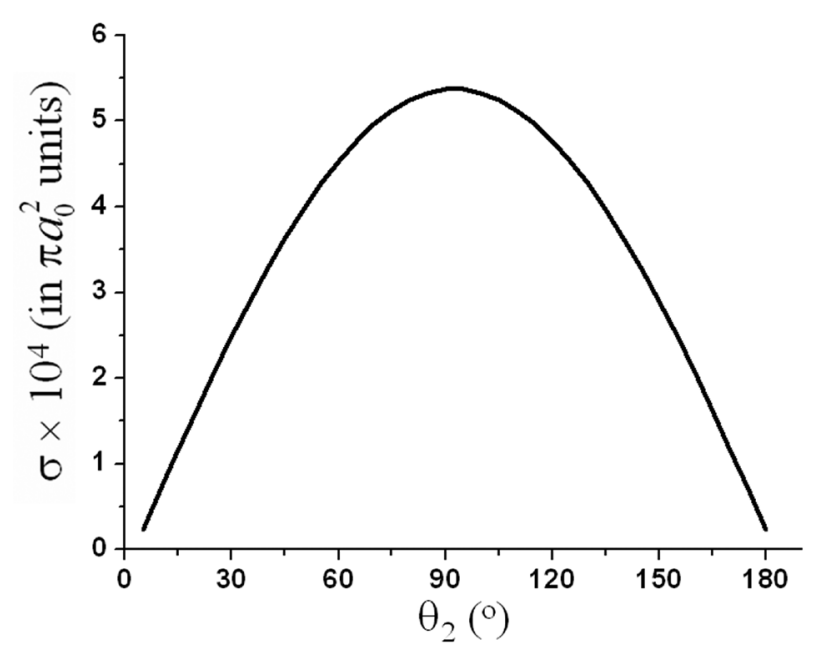

Figure 3. Angular $\left(\theta_{2}\right)$ dependence of the energy loss total cross section $(\sigma)$ for an incident energy $(\mathrm{E})$ of $1000 \mathrm{eV}$ with aperture angular resolutions of $\Delta \phi=5^{\circ}$ (fixed) and $\Delta \theta=5^{\circ}$ with $\theta$ varying from $0^{\circ}$ to $180^{\circ}$.

quantifying the sharing of electrons in a chemical bond. In addition to the previously analyzed oscillator strengths and Raman cross sections by the CBOP in heteropolar diatomic molecules, the results obtained in the present work, though for the particular case of the hydrogen molecule, indicate that electron energy-loss spectra may also provide useful information on the CBOP.

The hydrogen molecule, as a case study, has been particularly illustrative due to the quadrupole nature of the CBOP electron energy-loss cross section, which presents an impacting electron energy dependence and angular behavior distinguishable from the scattering cross sections for inter-band transitions, ionization and dissociation.

\section{Acknowledgements}

The authors are grateful for the financial support from the $\mathrm{CNPq}$ (Brazilian Agency) and from RENAMI and IMMC (Brazilian Scientific Programs).

\section{References}

1. Malta, O. L.; Batista, H. J.; Carlos, L. D.; Chem. Phys. 2002, 282, 21.

2. Malta, O. L.; Chem. Phys. Lett. 1982, 87, 27; 1982, 88, 353.

3. Albuquerque, R. Q.; Malta, O. L.; In Nato Science Series II: Math. Phys. Chem., vol. 126, Krupa, J. C.; Kulagin N. A. eds.; Kluwer Academic Publishers: Dordrecht, 2003, p. 141.

4. Albuquerque, R. Q.; PhD Thesis, Universidade Federal de Pernambuco, Brazil, 2004. (http://www.bdtd.ufpe.br/ tedeSimplificado//tde_busca/arquivo.php?codArquivo=2539)

5. Malta, O. L.; Chem. Phys. Lett. 2005, 406, 192.

6. Davydov, A. S.; Quantum Mechanics, $2^{\text {nd }}$ ed., Pergamon Press: Oxford, 1976.

7. Edmonds, A. R.; Angular Momentum in Quantum Mechanics, $2^{\text {nd }}$ ed., Princeton University Press: New Jersey, 1960.

8. Carter, C.; March, N. H.; Vincent, D.; Proc. Phys. Soc. (London) 1958, 71, 2.

9. Gallup, G. A.; Valence Bond Methods - Theory and Application, Cambridge University Press: New York, 2002.

10. McQuarrie, D. A.; Simon, J. D.; Physical Chemistry - A Molecular Approach, University Science Books: Sausalito, CA, 1997.

11. Magnasco, V.; J. Chem. Educ. 2008, 85, 1686.

12. Helgaker, T.; Jorgensen, P.; Olsen, J.; Molecular ElectronicStructure Theory, John Wiley \& Sons: New York, 2000.

13. Rescigno, T. N.; McCurdy Jr., C. W.; McKoy, V.; Phys. Rev. A: At., Mol., Opt. Phys. 1976, 13, 216.

14. Straube, H. C.; Renault, P.; Lindsay, B. G.; Smith, K. A.; Stebbings, R. F.; Phys. Rev. A: At., Mol., Opt. Phys. 1995, 54, 2146. 